\title{
An approach for the fatigue estimation of porous cast iron based on non-destructive testing results
}

\author{
André Heinrietz and Jan Hesseler \\ Fraunhofer Institute of Structural Durability and System Reliability LBF, Bartningstraße 47, \\ 64289 Darmstadt, Germany
}

\begin{abstract}
Big cast iron components made of spheroidal cast iron allow constructing big structures such as stone mills, engine blocks or wind mills with acceptable expenses. Thus, in economically optimized cast processes pores cannot be always prevented in thick walled cast iron components and these components are often rejected because of safety reasons. On the one hand the fatigue performance of high loadable spheroidal cast iron components is reduced significantly by the presence of local porosities which has been pointed out in the past. On the other hand concepts for the fatigue estimation based on fracture mechanics which take the size and localization of the defect into account can lead to erroneous estimations because the defect is modelled as a crack. The challenge of an estimation method is to derive a fatigue life without the necessity to perform component tests. In the contribution an estimation method is presented which is able to determine the fatigue strength of a material volume taking the pores into account. The method can be applied based on data from computer tomographic X-ray (CT) or Sampling Phased Array (SPA) ultrasonic analyses. The method is presented for three spheroidal cast iron types: ferritic GJS-400-18, ferritic GJS-450-15 with high silicon content and perlitic GJS-700-3.
\end{abstract}

\section{Fatigue strength of spheroidal cast iron}

In the past the dependencies of the S-N curves of spheroidal cast iron from graphite structure [1], macro porosity [2], micro porosity [3, 4] and non-metallic inclusions [2] have been observed. If no porosity and non-metallic inclusions are present the fatigue strength is ruled by micro-porosity or graphite structure depending on the amount of micro-porosity. Anyway, the amount of micro-porosity and the graphite structure are both depending on the inoculation method and the inoculant.

The plate material from which the specimens for tests on pore free material were extracted (Table 1) had been scanned for pores with ultrasonic and X-ray examinations. Based on the S-N curves of the pore free material (Table 1) the specimens containing pores are evaluated. Therefore all single results of the tests are shifted with means of the S-N curve parameter to a defined cycle number, exemplary shown in Fig. 1 (left, red triangles). The results show ruptures of specimens with pores beyond the kneepoint of S-N curves of pore-free material.

Because of these tests results a virtual kneepoint of S-N curves is recommended for further calculative assessments of porous material's fatigue strength with $\mathrm{N}=5 * 10^{6}$.

This is an Open Access article distributed under the terms of the Creative Commons Attribution License 4.0, which permits unrestricted use, distribution, and reproduction in any medium, provided the original work is properly cited. 


\section{MATEC Web of Conferences}

Table 1. Parameters of S-N curves of pore free material derived from tests [5].

\begin{tabular}{|l|c|c|c|c|}
\hline Material & $\begin{array}{c}\text { Fatigue strength } \\
\text { at kneepoint [MPa] }\end{array}$ & $\begin{array}{c}\text { Slope of } \\
\text { S-N curve }\end{array}$ & $\begin{array}{c}\text { Cycle number } \\
\text { at kneepoint }\end{array}$ & $\begin{array}{c}\text { Spread T } \\
\text { of SN, 10\%/90\% } \\
\text { SN curve }\end{array}$ \\
\hline GJS 400-18 & 143 & 6.7 & $1.58 * 10^{6}$ & $1: 1.19$ \\
\hline GJS 450-15 & 175 & 7.7 & $2 * 10^{6}$ & $1: 1.16$ \\
\hline GJS 700-2 & 190 & 5.6 & $1 * 10^{6}$ & $1: 1.23$ \\
\hline
\end{tabular}
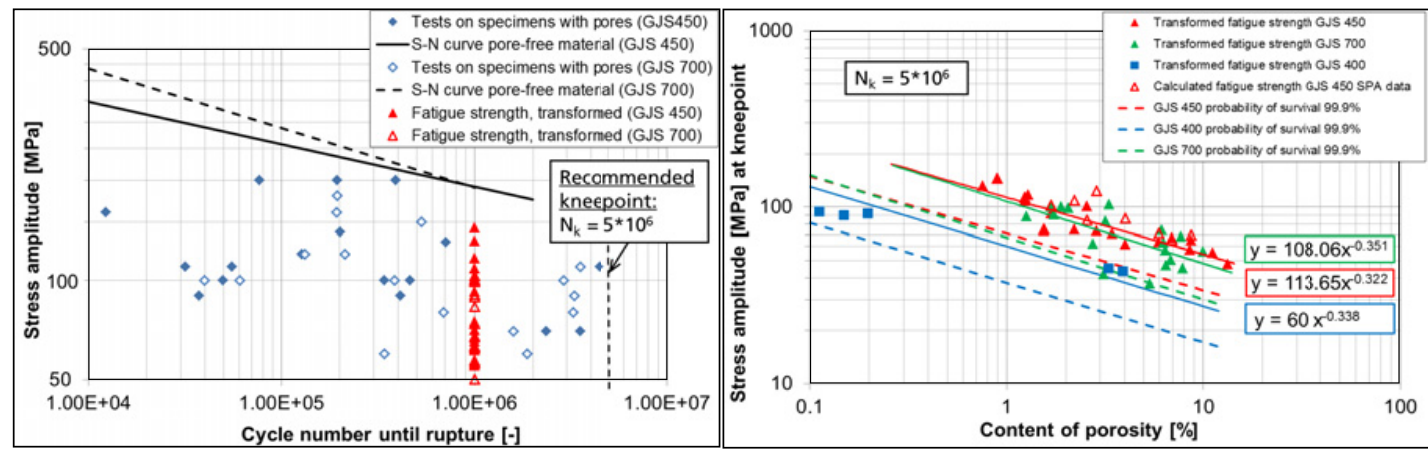

Figure 1. Results of tests on specimens with pores [5] and fatigue strengths transformed to a cycle number $\mathrm{N}=10^{6}$ (exemplary, left image); dependency of fatigue strength of GJS 400, GJS 450 and GJS 700 on porosity volume content; fatigue strengths are shown for a suggested virtual kneepoint of $\mathrm{N}=5 * 10^{6}$ (right image).

For calculative assessments of fatigue strength of porous cast iron based on non-destructive test results correlation functions of fatigue strength depending on selected variables are suggested. In Fig. 1, right a simple correlation model is shown which represents the dependency of fatigue strength on the porosity content. For this correlation model an exponential dependency of fatigue strength on the porosity content was estimated (1). In this model the fatigue strength can be calculated by the pore volume content $\mathrm{p}$ in percent. The coefficient $\mathrm{C}$ and the exponent $\mathrm{s}$ which represents the slope of the correlation curve are material specific values. The differences between the slopes s for GJS 450 (solid red line) and GJS 700 (solid green line) are small (Fig. 1).

$$
\sigma_{\text {fatigue }}=C_{\text {material }} \cdot \mathrm{p}^{-\mathrm{s}} \text {. }
$$

Because of the fact that only a few test results were carried out on specimens of GJS 400 the correlation function had to be estimated for GJS 400 by the experimental fatigue level of GJS 400 specimens. For this estimation the slope of the GJS 400 correlation function was estimated by the average slope of GJS 450 and GJS 700. The resulting correlation curve is shown in Fig. 1, right, solid blue line.

A single-variable correlation dependency may exclude systematic influence factors which impact the fatigue strength. Anyway, for the correlation functions the deviations are treated as scatter. It is clear that a distribution function for statistical evaluation cannot be confirmed by such a low number of tests performed (Fig 1, right). Assuming a log-normal distribution spreads of $\mathrm{T}_{\text {surv, } 10 \% / 90 \%}<1: 1.4$ (Fig. 1) are derived for the test series on porous specimens, postulating correlation functions of GJS 450 and GJS 700 (1).

\section{Derivation of porosities in specimens}

In order to get localized fatigue strengths for further calculations without the necessity to carry out fatigue tests on specimens non-destructive test data have to be interpreted. For big iron cast components 


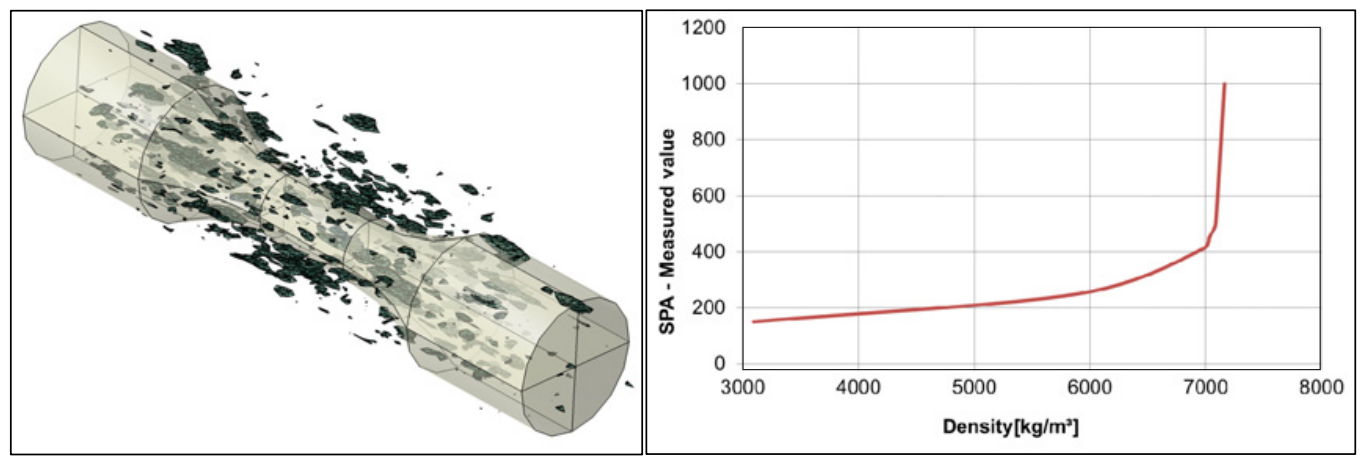

Figure 2. Virtual porosities as basis for 3D section parameter extraction (left); SPA transfer function for density.

ultra-sonic analyses is an applicable testing method. During methodology development CT X-ray analyses have been used to calibrate three dimensional sampling phased array (SPA) ultrasonic test results. The CT analyses were carried out for the test sections of the specimens tested in fatigue test (Fig. 2, right) with a resolution of $50 \mu \mathrm{m}$. Additionally, the plates from which the specimens were cut were analysed by SPA tests. Next, pore content for each section was calculated from CT data. Because SPA delivered data from whole plates the test sections of the specimens were cut out virtually from SPA data sets (Fig. 2, left).

A transfer function was derived describing the dependency of the SPA measured values from pore content (Fig. 2, right). With means of this transfer function the porosity in the specimens was analysed. The results of the porosities calculated by SPA values for the test results are shown in Fig. 1, right (GJS 450 , red open triangles).

\section{References}

[1] Heinrietz, A., Eufinger, J.; Sobota, A.; Stets, W.: Non Homogenous Microstructure of Cast Iron Components - Challenge for Fatigue Evaluation of Non-Destructively Tested "Defect Free" Components; The Minerals, Metals \& Materials Society TMS, Annual Meeting, Orlando FL (2012)

[2] Kaufmann, H., "Zur schwingfesten Bemessung dickwandiger Bauteile aus GGG-40 unter Berücksichtigung gießtechnisch bedingter Gefügeungänzen”, Fraunhofer LBF research report FB214, Fraunhofer Institute of Structural Durability and System Reliability LBF (1998)

[3] Eufinger, J.; Heinrietz, A.; Bruder, T.; Hanselka, H.: "An engineering approach to fatigue analysis based on elastic-plastic fracture mechanics" Fatigue Fract Engng Mater Struct 36, 65-74, Wiley Publishing Ltd. (2011)

[4] Eufinger, J.; Heinrietz, A.; Bruder, T.; Hanselka, H.: Microstructure based fatigue analysis of cast components under variable amplitude loading, Mat.-wiss. u.Werkstofftech. 2011, 42, No. 10

[5] Bleicher, C.: Lunkerfest LBF report no. 281 441, part 1 and 2, Fraunhofer Institute of Structural Durability and System Reliability LBF, unpublished, (2014) 